\title{
Oropharyngeal swallow physiology and swallowing-related quality of life in underweight patients with concomitant advanced chronic obstructive pulmonary disease
}

This article was published in the following Dove Press journal:

International Journal of COPD

\author{
Kendrea L Garand' \\ Charlie Strange ${ }^{2}$ \\ Luca Paoletti ${ }^{2}$ \\ Theresa Hopkins-Rossabi ${ }^{3}$ \\ Bonnie Martin-Harris ${ }^{3}$ \\ 'Department of Speech Pathology \\ and Audiology, College of Allied \\ Health Professions, University of \\ South Alabama, Mobile, AL 36688, \\ USA; ${ }^{2}$ Division of Pulmonary, Critical \\ Care, and Sleep Medicine, College \\ of Medicine, Medical University of \\ South Carolina, Charleston, SC 29425, \\ USA; ${ }^{3}$ Department of Communication \\ Sciences and Disorders, School of \\ Communication, Northwestern \\ University, Evanston, IL 60208, USA
}

Correspondence: Kendrea L Garand Department of Speech Pathology and Audiology, University of South Alabama, 572I USA Drive North, HAHN III9, Mobile, AL 36688, USA

Tel + I 25I 4459275

Email garand@southalabama.edu
Background: Swallowing impairment (dysphagia) has been associated with COPD and may contribute to exacerbations of this chronic and progressive disease. Further, risk of mortality increases with concomitant presence of cachexia in the COPD population. The purpose of this prospective study was to depict oropharyngeal swallowing physiology in underweight patients with stable but advanced-stage COPD.

Patients and methods: Ten underweight patients with stable but advanced COPD underwent a modified barium swallow study. Analysis of oropharyngeal swallowing function was completed using the standardized Modified Barium Swallow Impairment Profile and the Penetration-Aspiration Scale. Scores from the Dysphagia Handicap Index and 10-item Eating Assessment Tool were collected to assess patient perception of swallowing difficulty. Findings were compared to age- and sex-matched healthy controls.

Results: Significantly higher MBSImP oral total scores $(P=0.007)$ were observed in COPD patients compared to matched controls, but no difference was observed in pharyngeal total scores $(P=0.105)$. Patients with COPD had significantly higher maximum PAS scores compared with controls $(P=0.030)$. There was no significant difference in EAT-10 or DHI scores between patients with COPD and controls ( $P=0.41$ and $P=0.08$, respectively).

Conclusion: Underweight patients with severe but stable COPD present with dysphagia that may not be recognized by the patient. Further investigation is needed to elucidate the interaction between the respiratory-swallowing systems, how muscular weakness may contribute to swallowing impairment, and responsiveness to swallowing treatment.

Keywords: COPD, swallowing impairment, MBSImP, dysphagia, underweight

\section{Introduction}

A chronic inflammatory lung disease, COPD is the third leading cause of death in the United States. ${ }^{1}$ Exacerbations of symptoms are typical and can be severe during disease progression, whereas contributing to repeated, costly hospitalizations and early mortality. ${ }^{2}$ The estimated medical cost of COPD in 2012 was over 32 billion dollars $^{3}$ and is expected to rise, given frequent hospital readmissions within 30 days after initial discharge. ${ }^{4}$

COPD disease severity and clinical manifestations vary greatly between patients and research cohorts that have studied the incidence and type of associated oropharyngeal swallowing impairment. Further, assessment methods and metrics to investigate oropharyngeal swallowing mechanism and dysfunction have varied. One study 
reported the incidence of dysphagia associated with COPD was $85 \%$, with laryngeal penetration and tracheal aspiration observed in $28 \%$ and $42 \%$ of subjects, respectively. ${ }^{5}$ Further, complications secondary to swallowing impairments, such as aspiration pneumonia, dehydration, and airway obstruction, have been shown to contribute to disease exacerbations. ${ }^{6}$ The negative effects of exacerbation on patient's quality of life, physical and physiological well-being, and early mortality ${ }^{2}$ highlight the potential impact that dysphagia may have on the natural course of COPD.

Ghannouchi et al completed a systematic literature review revealing differences between COPD and healthy age-matched controls that were observed on videofluoroscopic imaging. ${ }^{7}$ For example, prolonged airway closure and a lower laryngeal position were observed in COPD patients with moderate to very severe disease. ${ }^{8}$ It was postulated that the prolonged airway closure represents an underlying protective response to reduce the risk of airway invasion. ${ }^{9}$ Other observations include prolonged transit times for solid foods, longer laryngeal vestibule closure times during liquid swallows, ${ }^{9}$ delayed swallow initiation, ${ }^{5}$ and oropharyngeal residue. ${ }^{10}$ Additionally, the rate of tracheal aspiration of thin liquids observed on videofluoroscopy in COPD patients was higher, compared to age-matched controls $(25 \%$ vs $0.1 \%$, respectively). ${ }^{11}$ Robinson et al found a significantly higher risk of aspiration on bedside swallow evaluations in a COPD group (44\%) compared to a non-COPD hospitalized group (29\%). ${ }^{12}$ Although the non-COPD patients' videofluoroscopic results were not reported, $17 \%$ of the COPD patients aspirated on videofluoroscopy. ${ }^{12}$ An additional study using fiberoptic endoscopic evaluation of swallowing revealed that the majority of patients with COPD presented with pharyngeal residue ( $90 \%$ ), and $25 \%$ of the study sample had bolus airway invasion. ${ }^{10}$

In addition to oropharyngeal motor impairment, altered upper airway sensory mechanics in COPD may further contribute to penetration/aspiration. Evidence suggests that COPD patients have reduced laryngopharyngeal sensitivity, resulting in decreased measures to clear pharyngeal residue. ${ }^{13}$ Moreover, the COPD patient may be less likely to engage in productive coughing to expel ingested material from the airway.

Furthermore, dysphagia is known to have devastating effects on nutritional status, with approximately $25 \%$ of patients with COPD developing cachexia - a wasting syndrome associated with a $50 \%$ reduction in median survival. ${ }^{14}$ To date, the authors are not aware of any study that investigates the concomitant effects of low body mass index (BMI) on swallowing in patients with COPD.
The purpose of the present prospective cohort study was to describe oropharyngeal swallowing physiology in patients with advanced-stage COPD (Global Initiative for Chronic Obstructive Lung Disease [GOLD] stages 3 and $4^{15}$ ) with concomitant low BMI using standardized, validated, and reproducible metrics of swallowing impairment and bolus airway invasion. This is the only investigation known to the authors that attempts to define dysphagia in a population of COPD patients who were also underweight. Prior research has reported on BMI but not limited inclusion to those patients presenting with low BMI. ${ }^{916}$ Further, we detailed oral intake, patient-reported symptoms of swallowing impairment, and the impact of dysphagia on quality of life. These findings were compared to age- and sex-matched healthy controls retrieved from a large normative database.

\section{Patients and methods}

Each patient underwent a baseline modified barium swallow study (MBSS) as part of a clinical trial evaluating the effects of an investigational nutritive agent to treat low BMI in 12 patients with advanced but stable COPD. Key eligibility criteria included: 1) ages 30-80 years; 2) GOLD Stage 3 or Stage 4 ${ }^{15}$; and 3) low weight, defined as BMI below $22 \mathrm{~kg} / \mathrm{m}^{2}$. Exclusion criteria were: 1) current tobacco, alcohol, or illicit drug use; 2) unstable cardiovascular disease; 3) cognitive dysfunction; 4) non-COPD lung disease; 5) history of organ transplant; 6) history of lung cancer or any metastatic cancer; 7) HIV/AIDS; 8) taking an immunosuppressive agent; and 9) taking greater than $20 \mathrm{mg}$ of prednisone or equivalent corticosteroid.

Swallowing-related data from healthy controls were extracted from a large normative database as part of a separate study investigating effects of aging on swallowing function. A healthy participant was defined as an individual without current or history of dysphagia, upper aerodigestive tract surgical procedures, hiatal hernia, pulmonary disease (eg, COPD), head and neck cancer, and/or neurological disease (eg, stroke, Parkinson's disease). Further, he/she was able to drink thin liquids and eat solids as part of a regular diet and did not have a known allergy or dietary restriction for food or barium contrast materials used in study procedures. For each COPD patient, a maximum of four age-matched ( \pm 2 years) and sex-matched controls were extracted.

All informed consent procedures were in accordance with the ethical standards of the responsible committee on human experimentation. Written informed consent was obtained from all study patients and healthy controls. This study received Institutional Review Board Approval from 
Medical University of South Carolina (IRBs\# Pro0027289 [COPD] and Pro00011566 [healthy controls]). This study was registered as a clinical trial with the ClinicalTrials.gov Identifier of NCT02067377.

Videofluoroscopic imaging was obtained using continuous fluoroscopy, and high-resolution images were recorded at 30 frames (Digital Swallowing Workstation Model 7100, Kay Elemetrics Corp.) Standardized consistencies of commercially prepared barium (Varibar $\left.{ }^{\circledR}\right)$ of increasing volume (5 mL, single-cup swallow, and sequential swallows of liquid) and viscosity (thin, nectar- and honey-thickened liquids, pudding, and cookie) were provided for a maximum of 12 swallowing tasks in two viewing planes (ten swallows lateral and two swallows anteroposterior) following the standardized Modified Barium Swallow Impairment Profile ${ }^{\mathrm{TM}}$ (MBSImP) approach. ${ }^{17}$

The MBSImP measures 17 validated and reliable components of swallow physiology across three functional domains: oral, pharyngeal, and esophageal. ${ }^{17}$ The operational definitions for each component score represent unique and unambiguous observations of structural movement, bolus flow, or both. ${ }^{17}$ The highest (worst) score across all swallowing tasks contributes to an overall impression (OI) score for each of the 17 components. A measure of overall oral impairment (oral total [OT]) is constructed on the basis of the sum score of components one through six OI scores (range 0-22). Likewise, overall pharyngeal impairment (pharyngeal total [PT]) is constructed on the basis of the sum of components seven through 16 OI scores (range $=0-29$ ). Scoring of videofluoroscopic studies were randomized among four speech-language pathologists with expertise in MBSImP interpretation method ( $\geq 80 \%$ inter-rater reliability).

The presence and degree of airway invasion were measured using the validated Penetration-Aspiration Scale (PAS) - an eight-point ordinal scale..$^{18} \mathrm{~A}$ score of 1 represents the absence of airway invasion, and a score of 8 represents an absent attempt to react to or expectorate aspirated material (ie, silent aspiration). ${ }^{18}$ Scores of 3 and greater are considered impaired, on the basis of normative data. ${ }^{19} \mathrm{PAS}$ was derived for each swallowing task.

The Functional Oral Intake Scale (FOIS) was used to document the patients' oral intake level. ${ }^{20,21}$ The FOIS consists of seven levels to assess diet level of safe oral intake, with Level 1 representing no oral intake and Level 7 representing total oral intake without restrictions. Additionally, each patient completed the Dysphagia Handicap Index $(\mathrm{DHI})^{22}$ and 10-item Eating Assessment Tool (EAT-10). ${ }^{20}$ The DHI measures the handicapping effects of dysphagia on emotional, functional, and physical aspects of the lives of dysphagic patients. ${ }^{22}$ Each of the 25 statements distributed across the three domains (emotional, functional, and physical) are rated as "never", "sometimes", or "always" occurring. Higher scores indicate more severe impairment. The DHI was validated in a heterogeneous dysphagic patient sample $(\mathrm{N}=77)$, using a control of non-dysphagic patients for clinical validity. The EAT-10 measures symptom-specific outcomes of dysphagia using ten items rated on a Likert scale of 0 (no problem) to 4 (severe problem), which was validated using a large cohort of voice and swallowing patients. The EAT-10 was validated using a large cohort of voice and swallowing patients $(\mathrm{N}=235)$, with a score of score of 3 and greater indicating abnormal swallowing function based on published normative data. ${ }^{20}$

COPD assessments included spirometry before and after bronchodilator, plethysmographic lung volumes to record the residual volume/total lung capacity (RV/TLC) ratio. A 6MWD to define the distance walked by standardized protocol. ${ }^{23}$ The St Georges Respiratory Questionnaire (SGRQ) was administered to define COPD-specific quality of life by total score. ${ }^{24}$ The SGRQ is used in patients with obstructive airways disease such as COPD to measure overall health and perceived well-being. It consists of 50 questions that cover symptom frequency and severity as well as activities that are limited by breathlessness. Scores range from 0 to 100 , with higher scores indicating greater limitation. The SGRQ has been used since 1991 and has been validated for correlations between symptoms and activity levels.

\section{Data collection}

MBSImP component scores of 1 and greater were considered impaired for purposes of analysis. However, lip closure, tongue base retraction, and pharyngeal residue were deemed impaired if the score was greater than 2 (scores of 1 on these components could be considered normal variance). Descriptive measures were calculated for MBSImP component and composite scores (oral and pharyngeal totals), PAS, DHI, and EAT-10. Mann-Whitney $U$ tests were calculated to compare composite scores between patients with COPD to sex- and age-matched ( \pm 2 years) healthy, non-dysphagic controls that were collected as part of a large normative study $(n=195)$ separate from the current project. ${ }^{25}$ After all the eligible control matches were selected, a maximum of four were randomly chosen for a total of 37 control subjects because one COPD subject only had one matching healthy control.

Regression analysis was conducted between measures of OT and PT scores and the following COPD physiologic 
tests: the RV/TLC ratio, 6MWD, and SGRQ. Study data were managed using REDCap. ${ }^{25}$

\section{Results}

Results of ten patients (five females) with a mean age of $62( \pm 8)$ years are presented. Two patients were removed from analysis because of previous anterior cervical surgeries which may impact swallowing function. All patients had obstructive lung disease, with most classified as Stage 4 (90\%). Thirtyseven adults (20 females) comprised the control group, with a mean age of $61( \pm 8)$ years. Further demographic and clinical characteristics are described in Table 1.

\section{MBSImP component and composite scores}

All patients demonstrated impairment in oral residue, initiation of pharyngeal swallow, tongue base retraction, pharyngeal residue, and esophageal clearance. Moreover, the majority of patients demonstrated impairment in bolus preparation/mastication and pharyngoesophageal segment opening (70\% and 90\%, respectively; Table 2 ).

The mean $( \pm \mathrm{SD})$ OT and PT scores were $7.7( \pm 2.6)$ and $7.9( \pm 3.0)$, respectively (Table 3 ). When composite scores of the patients with COPD were compared with matched controls, there was a significant difference in OT scores ( $P=0.007)$ but not PT scores $(P=0.105$; Table 3$)$.

Table I Patient demographics. Data presented as mean \pm SD unless otherwise reported

\begin{tabular}{|c|c|c|}
\hline Variables & $\begin{array}{l}\text { COPD } \\
(\mathbf{N}=10)\end{array}$ & $\begin{array}{l}\text { Heathy } \\
\text { controls }^{a} \\
(\mathbf{N}=37)\end{array}$ \\
\hline \multicolumn{3}{|l|}{ Age (years) } \\
\hline Mean \pm SD & $61.8 \pm 8.2$ & $61.0 \pm 7.8$ \\
\hline Range & $53-76$ & $5 \mathrm{I}-78$ \\
\hline Sex frequency (female \%) & $5(50.0 \%)$ & $20(54.1 \%)$ \\
\hline \multicolumn{3}{|c|}{ Racial category frequency (percentage) } \\
\hline White/Caucasian & $9(90 \%)$ & $32(86.5)$ \\
\hline Black/African American & I (I0\%) & $4(10.8)$ \\
\hline \multicolumn{3}{|c|}{ Ethnicity category frequency (percentage) } \\
\hline Non-Hispanic/non-Latino & $10(100 \%)$ & $36(97.3)$ \\
\hline \multirow[t]{2}{*}{$\mathrm{BMI}$} & $18.5 \pm 1.4$ & - \\
\hline & \multicolumn{2}{|c|}{ Range: 16.6-20.5 } \\
\hline \multicolumn{3}{|l|}{ Pulmonary function } \\
\hline FEV $\%$ predicted & $27.9 \pm 7.8$ & - \\
\hline FVC\% predicted & $70.0 \pm 10.9$ & - \\
\hline $\mathrm{FEV}_{1} / \mathrm{FVC}$ & $30.6 \pm 6.8$ & - \\
\hline TLC\% predicted & $125.1 \pm 31.9$ & - \\
\hline RV\% predicted & $202.1 \pm 76.1$ & - \\
\hline RV/TLC & $58.4 \pm 13.4$ & - \\
\hline Hand grip strength & $59.6 \pm 20.5$ & - \\
\hline \multicolumn{3}{|c|}{ 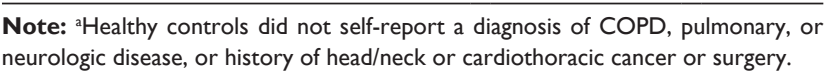 } \\
\hline \multicolumn{3}{|c|}{$\begin{array}{l}\text { Abbreviations: BMI, body mass index; } \mathrm{FEV}_{1} \text {, forced expiratory volume in the first } \\
\text { second; FVC, forced vital capacity; TLC, total lung capacity; RV, residual volume. }\end{array}$} \\
\hline
\end{tabular}

\section{PAS}

The mean PAS score $( \pm \mathrm{SD})$ for all COPD patients across swallowing tasks was $1.3( \pm 0.9)$, with a range of $1-8$ (Tables 3 and 4). Highest PAS scores were observed on the sequential thin liquid (worst score of 4 ) and sequential nectar-thickened liquid (worst score of 8) swallowing tasks. Patients with COPD had significantly higher maximum (worst) PAS scores compared with controls $(P=0.030)$ (Tables 3 and 4). One COPD patient was once observed to have silent aspiration (PAS=8) during the sequential nectarthickened liquid swallowing task only. Reduced laryngeal elevation and laryngeal vestibular closure contributed to the bolus entering the airway without clearance, which resulted in the residue falling below the true vocal folds on a subsequent swallow without patient attempt to expectorate the aspirated material.

\section{FOIS}

All patients had a FOIS level of 7, which indicates they reported a total oral diet of all consistencies without any restrictions.

\section{EAT-IO}

The EAT-10 mean $( \pm \mathrm{SD})$ total score was $2.5( \pm 3.2)$, with a range from 0 to 8 (Table 3 ). Forty-four percent of patients with COPD had a total score of 3 or greater as compared with $19 \%$ of healthy controls $(n=7)$. There was no significant difference in EAT-10 total scores between patients with COPD and controls $(P=0.413)$.

\section{DHI}

The mean $( \pm \mathrm{SD})$ total DHI score was $12.2( \pm 11.1)$, with a range from 2 to 36 (Table 3 ). Summary statistics across the physical, functional, and emotional domains are provided in Table 3. Comparisons between patients with COPD and controls $(\mathrm{n}=5)$ did not reveal a significant difference in DHI scores $(P=0.075)$.

\section{COPD metrics}

Correlations between the OT score trended with the RV/TLC ratio - a measure of hyperinflation and 6MWD distance - an important functional outcome in COPD. Further, the RV/ TLC trended with the component score bolus preparation/ mastication - a critical component of efficient bolus preparation and airway protection. PT scores trended with the SGRQ to suggest that both oral and pharyngeal components of swallowing are dysfunctional in severe COPD patients who perceive they have no swallowing problems (Figure 1). 
Table 2 MBSImP component OI scores. Summary measures are frequency (\%)

\begin{tabular}{|c|c|c|c|c|}
\hline \multirow[t]{3}{*}{ MBSImP component } & \multicolumn{2}{|l|}{ OI $=0$} & \multicolumn{2}{|l|}{ OI $\geq \mathbf{I}$} \\
\hline & COPD & Healthy & COPD & Healthy \\
\hline & $\mathbf{N}=10$ & $\mathbf{N}=\mathbf{3 7}$ & $\mathbf{N}=10$ & $\mathbf{N}=\mathbf{3 7}$ \\
\hline $\mathrm{Cl}$ - Lip closure ${ }^{\mathrm{a}}$ & $8(80 \%)$ & $34(92 \%)$ & $2(20 \%)$ & $3(8 \%)$ \\
\hline C2 - Bolus hold & $5(50 \%)$ & 22 (59\%) & $5(50 \%)$ & $15(4 \mid \%)$ \\
\hline C3 - Bolus preparation/mastication & $3(30 \%)$ & 33 (89\%) & $7(70 \%)$ & $4(11 \%)$ \\
\hline C4 - Lingual transport & 7 (70\%) & 27 (73\%) & $3(30 \%)$ & $10(27 \%)$ \\
\hline C5 - Oral residue & $0(0 \%)$ & $12(32 \%)$ & $10(100 \%)$ & $25(68 \%)$ \\
\hline C6 - Initiation of pharyngeal swallow & $0(0 \%)$ & $2(5 \%)$ & $10(100 \%)$ & 35 (95\%) \\
\hline C7 - Soft palate elevation & $5(50 \%)$ & $37(100 \%)$ & $5(50 \%)$ & $0(0 \%)$ \\
\hline C8 - Laryngeal elevation & $6(60 \%)$ & $16(43 \%)$ & $4(40 \%)$ & $21(57 \%)$ \\
\hline C9 - Anterior hyoid excursion & 7 (70\%) & $6(16 \%)$ & $3(30 \%)$ & 31 (84\%) \\
\hline ClO - Epiglottic movement & 7 (70\%) & $29(79 \%)$ & $3(30 \%)$ & $8(21 \%)$ \\
\hline CII - Laryngeal vestibular closure & $5(50 \%)$ & $31(84 \%)$ & $5(50 \%)$ & $6(16 \%)$ \\
\hline $\mathrm{Cl} 2$ - Pharyngeal stripping wave & $7(70 \%)$ & $22(59 \%)$ & $3(30 \%)$ & $15(41 \%)$ \\
\hline $\mathrm{Cl} 3$ - Pharyngeal contraction & $8(80 \%)$ & $30(81 \%)$ & $2(20 \%)$ & $7(19 \%)$ \\
\hline $\mathrm{Cl} 4$ - Pharyngoesophageal segment opening & $\mathrm{I}(10 \%)$ & $2(5 \%)$ & $9(90 \%)$ & $35(95 \%)$ \\
\hline $\mathrm{CI} 5$ - Tongue base retraction ${ }^{\mathrm{a}}$ & $0(0 \%)$ & $17(46 \%)$ & $10(100 \%)$ & $20(54 \%)$ \\
\hline $\mathrm{Cl} 6$ - Pharyngeal residue ${ }^{\mathrm{a}}$ & $0(0 \%)$ & 15 (41\%) & $10(100 \%)$ & $22(59 \%)$ \\
\hline CI7 - Esophageal clearance ${ }^{b}$ & $0(0 \%)$ & $19(51 \%)$ & $9(100 \%)$ & $18(49 \%)$ \\
\hline
\end{tabular}

Notes: ${ }^{\mathrm{O}} \mathrm{Ol}$ score of I for this component is considered normal variation. Thus, $\mathrm{OI}$ scores of 0 and I were collapsed under the Ol=0 column. ${ }^{\mathrm{b}} \mathrm{Missing}$ one score for a COPD patient.

Abbreviations: OI, overall impression; MBSImP, Modified Barium Swallow Impairment Profile.

\section{Discussion}

The purpose of this cohort study was to describe oropharyngeal swallowing physiology in patients with stable, advanced-stage COPD and were underweight $(\mathrm{BMI}<22$ ). Oropharyngeal swallowing impairment was observed in all COPD patients. Oral impairment and PAS scores were significantly worse from those of age- and sex-matched healthy controls. Swallowing impairment, despite the minimal airway invasion observed in this cohort, contributes to increased aspiration and nutritional risks. The patients included in this sample were stable; however, during an acute exacerbation, these patients may not be able to protect their airway as efficiently. Further, if there is a progression of dysphagia severity, it may

Table 3 Comparison between COPD and sex- and age-matched ( \pm 2 years) healthy controls. Data presented as mean \pm SD unless otherwise reported

\begin{tabular}{llll}
\hline Outcome measure & $\begin{array}{l}\text { COPD } \\
(\mathbf{n}=\mathbf{1 0})\end{array}$ & $\begin{array}{l}\text { Healthy } \\
\text { controls } \\
(\mathbf{n}=\mathbf{3 7})\end{array}$ & P-value \\
\hline MBSImP composite score & $7.7 \pm 2.6$ & $5.2 \pm 2.1$ & 0.007 \\
$\quad$ Oral total & $7.9 \pm 3.0$ & $6.0 \pm 2.6$ & $\mathrm{NS}$ \\
$\quad$ Pharyngeal total & $1.3 \pm 0.9$ & $1.1 \pm 0.5$ & $\mathrm{NS}$ \\
Penetration-Aspiration Scale score & 8 & 5 & 0.030 \\
$\quad$ Highest score & $2.6 \pm 3.2^{\mathrm{a}}$ & $1.5 \pm 3.3$ & $\mathrm{NS}$ \\
EAT-I0 total score & $12.2 \pm 11.1$ & $3.6 \pm 4.1^{\mathrm{b}}$ & $\mathrm{NS}$ \\
\hline Dysphagia Handicap Index total score & & &
\end{tabular}

Notes: ${ }^{a} \mathrm{~N}=9$ for this measure. ${ }^{b} \mathrm{~N}=6$ for this measure.

Abbreviations: MBSImP, Modified Barium Swallow Impairment Profile; EAT-I0, 10-item Eating Assessment Tool; NS, nonsignificant. contribute to COPD severity and exacerbations. Moreover, increasing dysphagia severity may further exacerbate nutritional impairments in patients who are already underweight (low BMI), with detrimental effects on the body, including further loss of muscle mass and gastrointestinal dysfunction. The latter is of interest because impairment in esophageal clearance in the upright position was observed in all COPD patients but only half of the healthy control subjects.

Interestingly, patients with COPD have no specific neurological or structural etiology known to account for dysphagia. However, all study patients exhibited delayed initiation of the pharyngeal swallow and oropharyngeal residue, which may indicate muscular weakness and/or reduced sensation of upper aerodigestive tract structures involved in swallowing - specifically, the tongue and pharynx. For example, the majority of patients exhibited decreased bolus preparation, and all patients demonstrated reduced tongue base retraction, suggesting lingual weakness that further supports findings from previous studies. ${ }^{5,10}$ Generalized weakness present in the COPD population has been reported to be related, at least in part, to poor nutrition and reduced exercise tolerance. ${ }^{26}$ Further, specific sarcopenia (which affects $15 \%$ of patients with stable COPD) may occur in diffuse muscle groups ${ }^{27}$ and can affect muscles used during swallowing. In addition, discoordination of respiration with impaired swallowing has been implicated in patients with stable and acute exacerbations of COPD. ${ }^{28-30}$ Compromised 
Table 4 Categorized PAS scores across swallowing tasks. Summary measures are frequency (\%)

\begin{tabular}{|c|c|c|c|c|c|c|}
\hline \multirow{2}{*}{$\begin{array}{l}\text { Swallowing } \\
\text { task }\end{array}$} & \multicolumn{2}{|c|}{ PAS $=1$ or $2^{a}$} & \multicolumn{2}{|c|}{ PAS $=3-5$} & \multicolumn{2}{|c|}{ PAS $=6-8$} \\
\hline & $\begin{array}{l}\text { COPD } \\
(N=10)\end{array}$ & $\begin{array}{l}\text { Healthy } \\
(\mathbf{N}=37)\end{array}$ & $\begin{array}{l}\text { COPD } \\
(N=10)\end{array}$ & $\begin{array}{l}\text { Healthy } \\
(\mathrm{N}=37)\end{array}$ & $\begin{array}{l}\text { COPD } \\
(\mathrm{N}=10)\end{array}$ & $\begin{array}{l}\text { Healthy } \\
(N=37)\end{array}$ \\
\hline $5 \mathrm{~mL}$ thin 2 & $10(100 \%)$ & $36(97 \%)$ & $0(0 \%)$ & I (3\%) & $0(0 \%)$ & $0(0 \%)$ \\
\hline Cup thin & $10(100 \%)$ & $35(95 \%)$ & $0(0 \%)$ & $2(5 \%)$ & $0(0 \%)$ & $0(0 \%)$ \\
\hline Sequential thin & $8(80 \%)$ & 34 (92\%) & $2(20 \%)$ & $3(8 \%)$ & $0(0 \%)$ & $0(0 \%)$ \\
\hline $5 \mathrm{~mL}$ nectar & $10(100 \%)$ & 36 (97\%) & $0(0 \%)$ & I (3\%) & $0(0 \%)$ & $0(0 \%)$ \\
\hline Cup nectar & $10(100 \%)$ & 37 (100\%) & $0(0 \%)$ & $0(0 \%)$ & $0(0 \%)$ & $0(0 \%)$ \\
\hline Sequential nectar & $9(90 \%)$ & 37 (100\%) & $0(0 \%)$ & $0(0 \%)$ & I (10\%) & $0(0 \%)$ \\
\hline Honey & $10(100 \%)$ & 37 (100\%) & $0(0 \%)$ & $0(0 \%)$ & $0(0 \%)$ & $0(0 \%)$ \\
\hline Pudding & $10(100 \%)$ & 37 (100\%) & $0(0 \%)$ & $0(0 \%)$ & $0(0 \%)$ & $0(0 \%)$ \\
\hline Cookie & $10(100 \%)$ & $37(100 \%)$ & $0(0 \%)$ & $0(0 \%)$ & $0(0 \%)$ & $0(0 \%)$ \\
\hline
\end{tabular}

Notes: ${ }^{a}$ Data from Robbins. ${ }^{19}$ PAS scores of I or 2 are frequently observed in healthy adults and, thus, are considered "normal".

Abbreviation: PAS, Penetration-Aspiration Scale.

pulmonary function and impaired swallowing increase aspiration risk in patients with COPD.

Another contributor of dysphagia may be the reduction in oropharyngeal sensation. Similar to results by

A

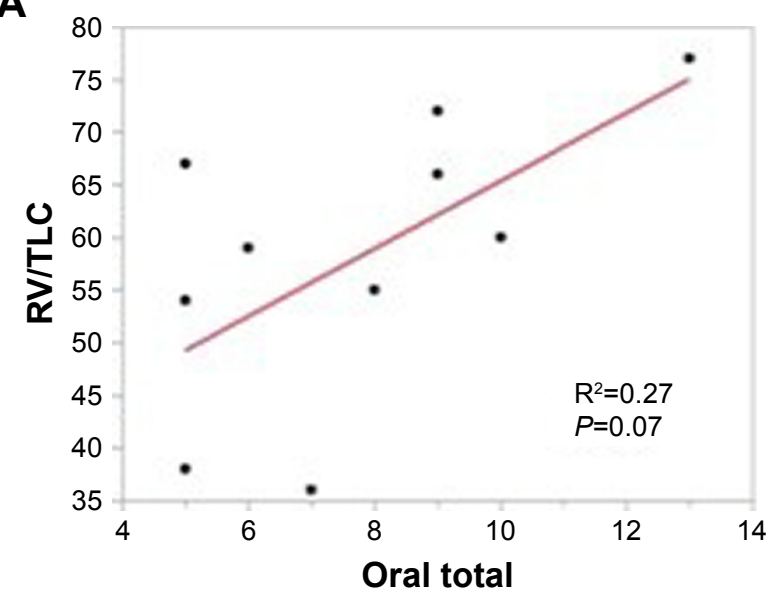

C

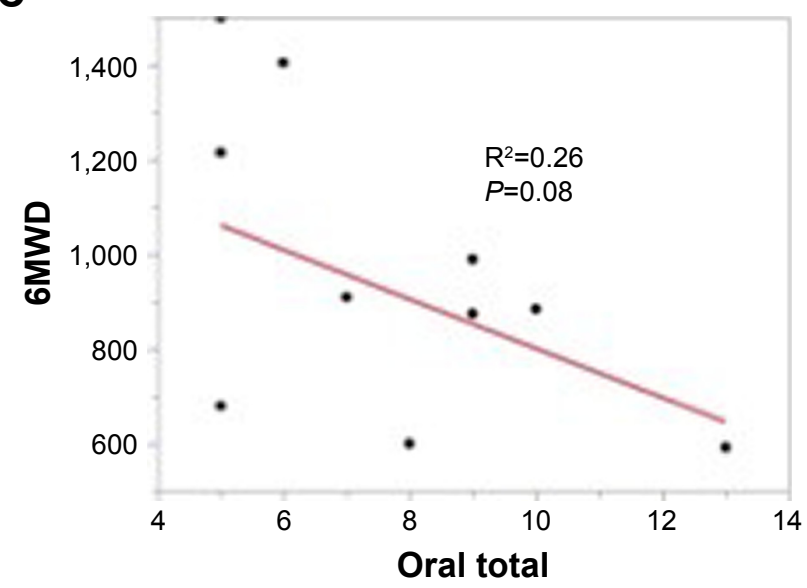

Good-Fratturelli et $\mathrm{al}^{5}$ who found delayed initiation of the pharyngeal swallow in $60 \%$ of patients, we found delayed swallowing in all patients in this COPD cohort. Notably, the pharyngeal swallow was not triggered until the leading

B

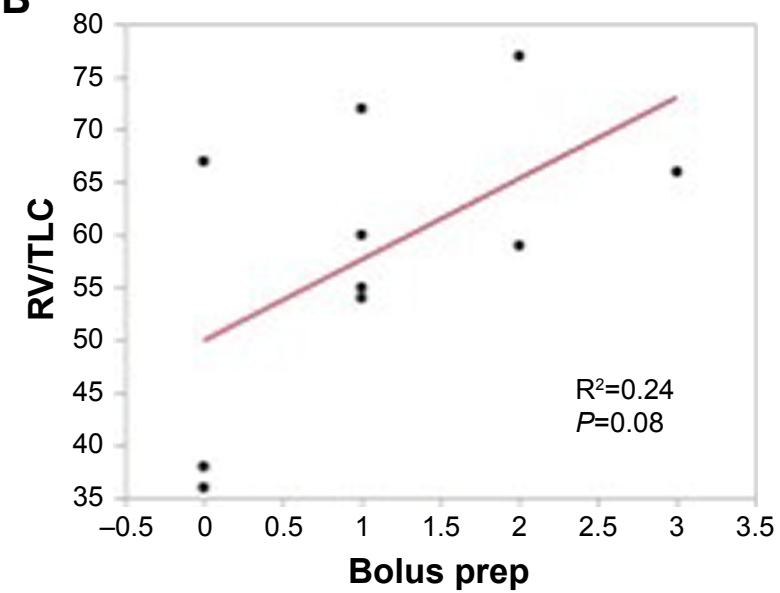

D

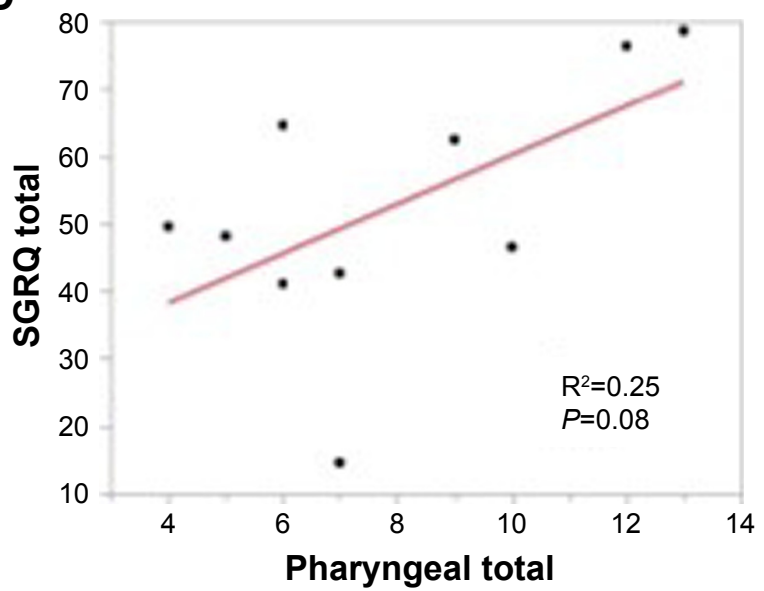

Figure I Analysis of COPD metrics oral total scores on the MBSImP are associated with measures of hyperinflation.

Notes: Oral total scores are associated with measures of hyperinflation $(\mathbf{A})$ and reflected in bolus preparation scores (B). Both oral and pharyngeal total scores are associated with measures of COPD severity including 6MWD (C) and SGRQ (D).

Abbreviations: RV/TLC, residual volume/total lung capacity; SGRQ, St Georges Respiratory Questionnaire; MBSImP, Modified Barium Swallow Impairment Profile. 
edge of the bolus was well into the pharynx in $60 \%$ of our patients on larger sequential swallowing tasks. Patients with COPD had consistently higher impairment rates for initiation of pharyngeal swallow as compared to matched controls, although differences on comparison were found to be non-significant. Two possible explanations for this result include the small sample size and the normal variation on this swallowing component observed in aging adults. For example, it has been shown that healthy older adults may initiate pharyngeal swallowing later when the leading edge of the bolus is advanced into the airway without aspiration occurrence. ${ }^{31}$ Interestingly, however, delayed swallow initiation was also observed in $88 \%$ of patients studied with recent exacerbation of COPD by Kobayashi et al. ${ }^{32}$ It may be that swallow delay may be confounded by other impaired airway protection mechanisms, such as decreased sensation, in patients with COPD. For example, Clayton et al reported decreased laryngeal sensation in patients with COPD, together with abnormal pharyngeal residue; the latter was observed in our cohort. ${ }^{13}$ The high incidence of oropharyngeal residue and delayed initiation of the swallow may in part result from overall reduced sensation. It has been proposed that the impact of corticosteroids, current or past smoking behavior, and oxygen therapy may impact pharyngeal and laryngeal mucosal sensory receptors of the pharyngeal and laryngeal mucosa in this population, and could then lead to decreased sensation. ${ }^{13}$

Impairment in laryngeal elevation was observed in almost half of the studied patients. Furthermore, those patients with decreased laryngeal elevation frequently presented with incomplete laryngeal vestibule closure, which further places patients at risk for airway invasion at the height of the swallow (when the vestibule should be fully closed and shielded from the bolus flowing through the pharynx). These findings support the investigation by Mokhlesi et al who also observed a lowered laryngeal position at this point in the swallow in patients with stable COPD patients. ${ }^{8}$

A higher incidence of laryngeal penetration and tracheal aspiration in COPD patients has been reported in previous studies, with the suggestion that ingested material in the airway may increase exacerbations in COPD. ${ }^{6}$ Good-Fratturelli et al reported $28 \%$ and $42 \%$ penetration and aspiration rates, respectively, ${ }^{5}$ similar to other studies. ${ }^{10-12}$ Although only one patient had one instance of silent aspiration, outside of the clinical context of the MBSS, this physiologic impairment during daily eating and drinking may well result in trace, low-grade aspiration and, therefore, confound pulmonary status.
The patients' perception of swallow function was incongruent with the nature and severity of the swallowing impairment observed. Their self-reported symptomatology related to swallowing impairment (EAT-10) and subsequent impact on quality of life (DHI) was minimal, with no difference observed between patient scores and matched healthy controls. Further, the patients in this cohort indicated no dietary restrictions (FOIS Level 7). Reduced pharyngeal sensation may account, in part, for the patient's limited insight into swallowing impairment. Further, reduced laryngopharyngeal sensation places the patient at increased risk for aspiration and aspiration-related pulmonary complications. The mismatch between patient's perceived and actual swallowing impairment and the nature of the swallowing impairments may signal a need for swallowing assessment and subsequent management in this patient population.

\section{Study limitations}

The primary limitation of this study includes the small patient sample representing a subset of COPD patients. The original sample size included 12 patients, but two were excluded secondary to history of anterior cervical discectomy fusion (ACDF) spinal surgeries because of the known swallowing impairment that may be result from disruption to the sensory end organs and muscles of the pharynx. Because this analysis was specifically investigating effects of COPD and underweight on swallowing function, we did not want potential confounders influencing results. Further, an MBSS represents only a few minutes in time. Fatigue is known to greatly impact patients with COPD when completing basic activities of daily living - including eating and drinking. ${ }^{33}$ It follows that swallowing function could likely further deteriorate outside the context of a clinical assessment and over the course of a full meal, leading to increased risk of aspiration. Next, certain measures (ie, BMI, hand grip strength, and spirometry) were not available in the normative dataset and, thus, we were not able to further analyze comparisons between the patient group and their matched controls. Lastly, we did not have data available for advanced-stage but stable COPD patients with normal weight for comparison. This would have strengthened clinical implications for the contribution of low BMI on swallowing function.

\section{Conclusion}

The results of this study suggest that patients with severe but stable COPD with concomitant underweight and dysphagia may require a multisystem approach for defining and 
rehabilitating dysphagia. Although the evidence from this current study revealed the presence of significant swallow impairment in underweight patients with severe COPD, there remains the lack of a clear cause and effect. This investigation included patients with a BMI below $22 \mathrm{~kg} / \mathrm{m}^{2}$. Future research should include patients with COPD who present with both higher and lower BMI to determine if factors such as overall body mass, rather than COPD status, impacts swallow function.

Swallowing impairment in COPD is likely a multifactorial issue and influenced by muscular weakness, alteration in oropharyngeal and laryngeal sensation, and mismatch between patient perception and actual presence and severity of dysphagia. Moreover, patients with COPD have been shown to have disruption in the coordinative processes between respiration and swallowing. Discoordination between the respiratory cessation and swallowing initiation has been shown to contribute to dysphagia and aspiration. ${ }^{34}$ Further investigations that directly examine aberrations in respiratory swallowing coordination and oropharyngeal muscle function on the type and severity of dysphagia are warranted. Such studies will lead to the discovery of potential behavioral interventions that target the impaired mechanisms contributing to disruptions in swallowing safety and efficiency in patients with COPD.

\section{Acknowledgments}

The authors would like to acknowledge Julie Blair, MA, CCC-SLP, BCS-S, Kate Davidson, MS, CCC-SLP, and R Jordan Hazelwood, PhD, CCC-SLP, BCS-S, CBIS, for their assistance with data collection and analysis of videofluoroscopic examinations of oropharyngeal swallowing in this patient sample.

This work was partially supported by Entera Health, Inc. (grant no. EH5220; principal investigator [PI]: Paoletti), Veterans Affairs Career Development Award-1 (VA CDA-1) RR\&D (grant no. 1IK1RX001628-01A1; PI: Garand), the National Institute on Deafness and Other Communication Disorders of the National Institutes of Health (grant no. K24DC12801; PI: Martin-Harris), and the South Carolina Clinical \& Translational Research (SCTR) Institute, with an academic home at the Medical University of South Carolina, NIH/NCATS (grant no. TL1 TR000061; PI: Brady; Project PI: Garand), and the American Speech-Language-Hearing Foundation (PI: Garand).

This paper was presented as a scientific paper presentation at the 24th Annual Meeting of the Dysphagia Research Society, February 25-27, 2016, Tucson, AZ, USA. Link: https://c.ymcdn.com/sites/dysphagiaresearch.sitrym.com/ resource/resm//Meeting Files/DRS 2016 Annual Mtg and Post.pdf.

The paper's abstract was published in Dysphagia. 2016; Vol 31(6):803-804.

This paper was also presented as a Technical research presentation at the 2016 American Speech-Language-Hearing Association (ASHA) Annual Convention, November 17-19, 2016; Philadelphia, PA, USA. (ASHA is not an openly accessible published abstract).

\section{Disclosure}

CS has received grants and non-financial support from the Alpha-1 Foundation, CSL Behring, and Pulmonx; grants, personal fees, and non-financial support from Grifols; grants from Inhibrx, Novartis, Pulmonx, and Shire; and non-financial support from Abeona and personal fees and non-financial support from AstraZeneca, all outside the submitted work. BMH receives copyright royalties from Northern Speech Services and Medical University of South Carolina and is the principal investigator (NIH/NIDCD, 1K24DC12801) in a research study and mentoring of swallowing impairment and respiratory-swallow coordination (2013-2018). KLG, THR, and LP report no conflicts of interest in this work.

\section{References}

1. Kochanek KD, Murphy SL, Xu J, Tejada-vera B. Deaths: Final Data for 2014. Natl Vital Stat Rep. 2016;65(4):1-122.

2. Anzueto A. Impact of exacerbations on COPD. Eur Respir Rev. 2010; 19(116):113-118.

3. Ford ES, Murphy LB, Khavjou O, Giles WH, Holt JB, Croft JB. Total and state-specific medical and absenteeism costs of COPD among adults aged $\geq 18$ years in the United States for 2010 and projections through 2020. Chest. 2015;147(1):31-45.

4. Jencks SF, Williams MV, Coleman EA. Rehospitalizations among patients in the Medicare fee-for-service program. N Engl J Med. 2009; 360(14):1418-1428.

5. Good-Fratturelli MD, Curlee RF, Holle JL. Prevalence and nature of dysphagia in VA patients with COPD referred for videofluoroscopic swallow examination. J Commun Disord. 2000;33(2):93-110.

6. Steidl E, Ribeiro CS, Gonçalves BF, Fernandes N, Antunes V, Mancopes R. Relationship between Dysphagia and Exacerbations in Chronic Obstructive Pulmonary Disease: A Literature Review. Int Arch Otorhinolaryngol. 2015;19(1):74-79.

7. Ghannouchi I, Speyer R, Doma K, Cordier R, Verin E. Swallowing function and chronic respiratory diseases: Systematic review. Respir Med. 2016;117:54-64.

8. Mokhlesi B, Logemann JA, Rademaker AW, Stangl CA, Corbridge TC. Oropharyngeal deglutition in stable COPD. Chest. 2002; 121(2):361-369.

9. Cassiani RA, Santos CM, Baddini-Martinez J, Dantas RO. Oral and pharyngeal bolus transit in patients with chronic obstructive pulmonary disease. Int J Chron Obstruct Pulmon Dis. 2015;10:489-496.

10. Clayton NA, Carnaby GD, Peters MJ, Ing AJ. Impaired laryngopharyngeal sensitivity in patients with COPD: the association with swallow function. Int J Speech Lang Pathol. 2014;16(6):615-623. 
11. Cvejic L, Harding R, Churchward T, et al. Laryngeal penetration and aspiration in individuals with stable COPD. Respirology. 2011; 16(2):269-275.

12. Robinson DJ, Jerrard-Dunne P, Greene Z, Lawson S, Lane S, O’Neill D. Oropharyngeal dysphagia in exacerbations of chronic obstructive pulmonary disease. Eur Geriatr Med. 2011;2(4):201-203.

13. Clayton NA, Carnaby-Mann GD, Peters MJ, Ing AJ. The effect of chronic obstructive pulmonary disease on laryngopharyngeal sensitivity. Ear Nose Throat J. 2012;91(9):370, 372, 374 passim.

14. Wagner PD. Possible mechanisms underlying the development of cachexia in COPD. Eur Respir J. 2008;31(3):492-501.

15. Rabe KF, Hurd S, Anzueto A, et al. Global strategy for the diagnosis, management, and prevention of chronic obstructive pulmonary disease: GOLD executive summary. Am J Respir Crit Care Med. 2007;176(6): 532-555.

16. Chaves RD, Carvalho CR, Cukier A, Stelmach R, Andrade CR. Symptoms of dysphagia in patients with COPD. J Bras Pneumol. 2011;37(2): 176-183.

17. Martin-Harris B, Brodsky MB, Michel Y, et al. MBS measurement tool for swallow impairment-MBSImp: establishing a standard. Dysphagia. 2008;23(4):392-405.

18. Rosenbek JC, Robbins JA, Roecker EB, Coyle JL, Wood JL. A penetration-aspiration scale. Dysphagia. 1996;11(2):93-98.

19. Robbins J, Coyle J, Rosenbek J, Roecker E, Wood J. Differentiation of normal and abnormal airway protection during swallowing using the penetration-aspiration scale. Dysphagia. 1999;14(4):228-232.

20. Belafsky PC, Mouadeb DA, Rees CJ, et al. Validity and reliability of the Eating Assessment Tool (EAT-10). Ann Otol Rhinol Laryngol. 2008;117(12):919-924.

21. Crary MA, Mann GD, Groher ME. Initial psychometric assessment of a functional oral intake scale for dysphagia in stroke patients. Arch Phys Med Rehabil. 2005;86(8):1516-1520.

22. Silbergleit AK, Schultz L, Jacobson BH, Beardsley T, Johnson AF. The Dysphagia handicap index: development and validation. Dysphagia. 2012;27(1):46-52.

23. ATS statement, ATS Committee on Proficiency Standards for Clinical Pulmonary Function Laboratories, Statement ATS. ATS statement: guidelines for the six-minute walk test. Am J Respir Crit Care Med. 2002;166(1):111-117.
24. Jones PW, Quirk FH, Baveystock CM. The St George's Respiratory Questionnaire. Respir Med. 1991;85 Suppl B:25-31.

25. Harris PA, Taylor R, Thielke R, Payne J, Gonzalez N, Conde JG. Research electronic data capture (REDCap) - a metadata-driven methodology and workflow process for providing translational research informatics support. J Biomed Inform. 2009;42(2):377-381.

26. Maltais F, Decramer M, Casaburi R, et al. An official American Thoracic Society/European Respiratory Society statement: update on limb muscle dysfunction in chronic obstructive pulmonary disease. Am J Respir Crit Care Med. 2014;189(9):e15-e62.

27. Jones SE, Maddocks M, Kon SS, et al. Sarcopenia in COPD: prevalence, clinical correlates and response to pulmonary rehabilitation. Thorax. 2015;70(3):213-218.

28. Shaker R, Li Q, Ren J, et al. Coordination of deglutition and phases of respiration: effect of aging, tachypnea, bolus volume, and chronic obstructive pulmonary disease. Am J Physiol. 1992;263(5 Pt 1): G750-G755.

29. Gross RD, Atwood CW, Ross SB, Olszewski JW, Eichhorn KA. The coordination of breathing and swallowing in chronic obstructive pulmonary disease. Am J Respir Crit Care Med. 2009;179(7):559-565.

30. Terzi N, Normand H, Dumanowski E, et al. Noninvasive ventilation and breathing-swallowing interplay in chronic obstructive pulmonary disease*. Crit Care Med. 2014;42(3):565-573.

31. Martin-Harris B, Brodsky MB, Michel Y, Lee F-S, Walters B. Delayed initiation of the pharyngeal swallow: normal variability in adult swallows. J Speech Lang Hear Res. 2007;50(3):585-594.

32. Kobayashi S, Kubo H, Yanai M. Impairment of the swallowing reflex in exacerbations of COPD. Thorax. 2007;62(11):1017.

33. Cochrane WJ, Afolabi OA. Investigation into the nutritional status, dietary intake and smoking habits of patients with chronic obstructive pulmonary disease. J Hum Nutr Diet. 2004;17(1):3-11; quiz 13-15.

34. Martin-Harris B. Clinical implications of respiratory-swallowing interactions. Curr Opin Otolaryngol Head Neck Surg. 2008;16(3): 194-199.
International Journal of COPD

\section{Publish your work in this journal}

The International Journal of COPD is an international, peer-reviewed journal of therapeutics and pharmacology focusing on concise rapid reporting of clinical studies and reviews in COPD. Special focus is given to the pathophysiological processes underlying the disease, intervention programs, patient focused education, and self management protocols.

\section{Dovepress}

This journal is indexed on PubMed Central, MedLine and CAS. The manuscript management system is completely online and includes a very quick and fair peer-review system, which is all easy to use. Visit http://www.dovepress.com/testimonials.php to read real quotes from published authors. 